\title{
Recent results on spectator-induced electromagnetic effects in ultrarelativistic light- and heavy-ion collisions
}

\section{Iwona Sputowska*}

The Henryk Niewodniczański Institute of Nuclear Physics PAN, Kraków, Poland;

E-mail: iwona.sputowska@cern.ch

\section{Andrzej Rybicki}

The Henryk Niewodniczański Institute of Nuclear Physics PAN, Kraków, Poland;

E-mail: andrzej.rybickidcern.ch

\section{Antoni Szczurek}

The Henryk Niewodniczański Institute of Nuclear Physics PAN, Kraków, Poland;

E-mail: antoni.szczurek@ifj.edu.pl

\section{Mirek Kiełbowicz}

The Henryk Niewodniczański Institute of Nuclear Physics PAN, Kraków, Poland;

E-mail: miroslaw.kielbowicziefj.edu.pl

\section{Vitalii Ozvenchuk}

The Henryk Niewodniczański Institute of Nuclear Physics PAN, Kraków, Poland;

E-mail: vitalii.ozvenchukeifj.edu.pl

\begin{abstract}
This paper focuses on studies of spectator-induced electromagnetic (EM) effects on charged pion emission observed for three different colliding systems: $\mathrm{Pb}+$ gas, peripheral $\mathrm{Pb}+\mathrm{Pb}$ and $\mathrm{Ar}+\mathrm{Sc}$ collisions at CERN SPS energies. New data on Ar+Sc collisions from the NA61/SHINE experiment bring a first insight into EM effects in small systems. In this paper, we present the improvement of our phenomenological model description which takes into account spectator fragmentation as well as the possible influence of the net positive participant charge close to the spectator system.
\end{abstract}

European Physical Society Conference on High Energy Physics - EPS-HEP2019 -

10-17 July, 2019

Ghent, Belgium

\footnotetext{
*Speaker.
} 


\section{Introduction}

A moving charge generates an electromagnetic field. Recent studies [1] suggest that one of the direct consequences of this electromagnetic law gives rise to a spectator-induced electromagnetic (EM) effect in heavy-ion collisions. This latter phenomenon can be understood as Coulomb interaction between charged particles emitted from the participant zone and two positively charged nuclear remnants that did not participate in the collision process, called spectator systems (see Fig. $1)$.

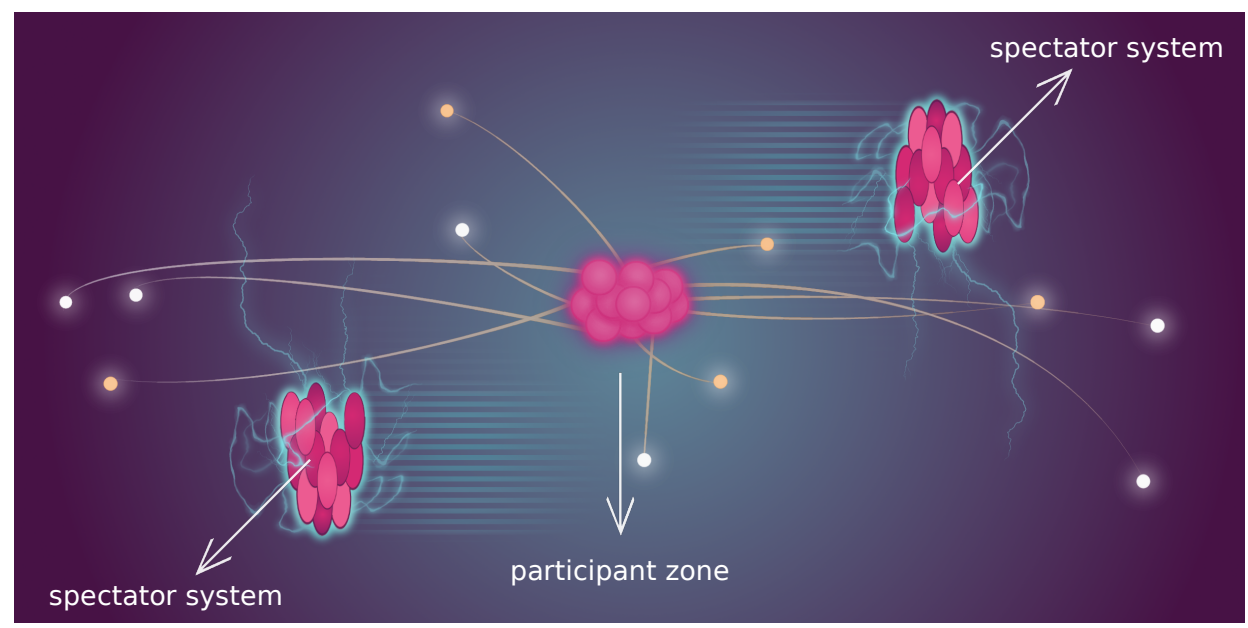

Figure 1: A graphic representation of a non-central nucleus-nucleus collision with a schematically outlined participant zone and two spectators systems.

The essence of the spectator-induced EM effect manifests itself in the observed change in the spectra of measured charged particles. It is induced by the electromagnetic field, generated by the highly charged spectator systems moving in the opposite direction with relativistic speed, acting on trajectories of charged particles produced in the non-central heavy-ion collision. Interest in the study on EM effects arise mostly from recent findings $[1,2,3,4]$ that have shown the spectator-induced electromagnetic effect is sensitive to the space-time evolution of the system and can become a new source of information in that matter.

So far, the research on that subject has been mostly restricted to the analysis of large colliding systems like peripheral $\mathrm{Pb}+\mathrm{Pb}$ or $\mathrm{Pb}+$ gas reactions [5] at the SPS (Super Proton Synchrotron) energies at CERN. However, this paper provides a general overview of the results measured on spectator-induced EM phenomena for both light- and heavy-ion collisions. The main focus is placed on analysis on new data on Ar+Sc collisions from the NA61/SHINE Collaboration which provides the first investigation of the spectator-induced EM effect in small systems at the SPS energies. This work discusses also the implications of the evolution of the spectator system itself on the measured spectra of charged $\pi$ mesons.

\section{EM effects in ultra-relativistic light- and heavy-ion collisions}

\subsection{Comparison of the $\mathrm{EM}$ effect in peripheral $\mathrm{Pb}+\mathrm{Pb}, \mathrm{Pb}+\mathrm{gas}$ and $\mathrm{Ar}+\mathrm{Sc}$ collisions}

Figure 2 (a)-(d) juxtapose experimental data of the spectator-induced EM effect observed in 
$\pi$ meson spectra obtained in heavy-ion collisions of $\mathrm{Pb}+$ gas and peripheral $\mathrm{Pb}+\mathrm{Pb}$ reactions with new results measured in light-ion reactions such as intermediate and central $\mathrm{Ar}+\mathrm{Sc}$ interactions. All four panels in Figure 2 show the $\pi^{+}$over $\pi^{-}$ratio plotted as a function of Feynman variable $x_{F}{ }^{1}$ for selected values of transverse momentum $p_{\mathrm{T}}$, both variables $x_{F}$ and $p_{\mathrm{T}}$ were considered in collision c.m.s. Presented results were obtained in the projectile fragmentation region $\left(x_{F}>0\right)$.

Data for asymmetric $\mathrm{Pb}+$ gas and symmetric peripheral $\mathrm{Pb}+\mathrm{Pb}$ collisions were measured at $\sqrt{s_{\mathrm{NN}}}=17.3 \mathrm{GeV}$ in the fixed target NA49 experiment (at the CERN SPS). In this paper, the term $\mathrm{Pb}+$ gas reactions should be interpreted as the collisions of the large lead projectile with smaller nuclei of gas $(\mathrm{Pb}+\mathrm{N}, \mathrm{Pb}+\mathrm{O})$ in the vicinity of NA49 target (see [5]). New results on $\mathrm{Ar}+\mathrm{Sc}$ collisions were obtained in the NA61/SHINE fixed target experiment at CERN SPS at energy $\sqrt{s_{\mathrm{NN}}}=16.8$ $\mathrm{GeV}$.
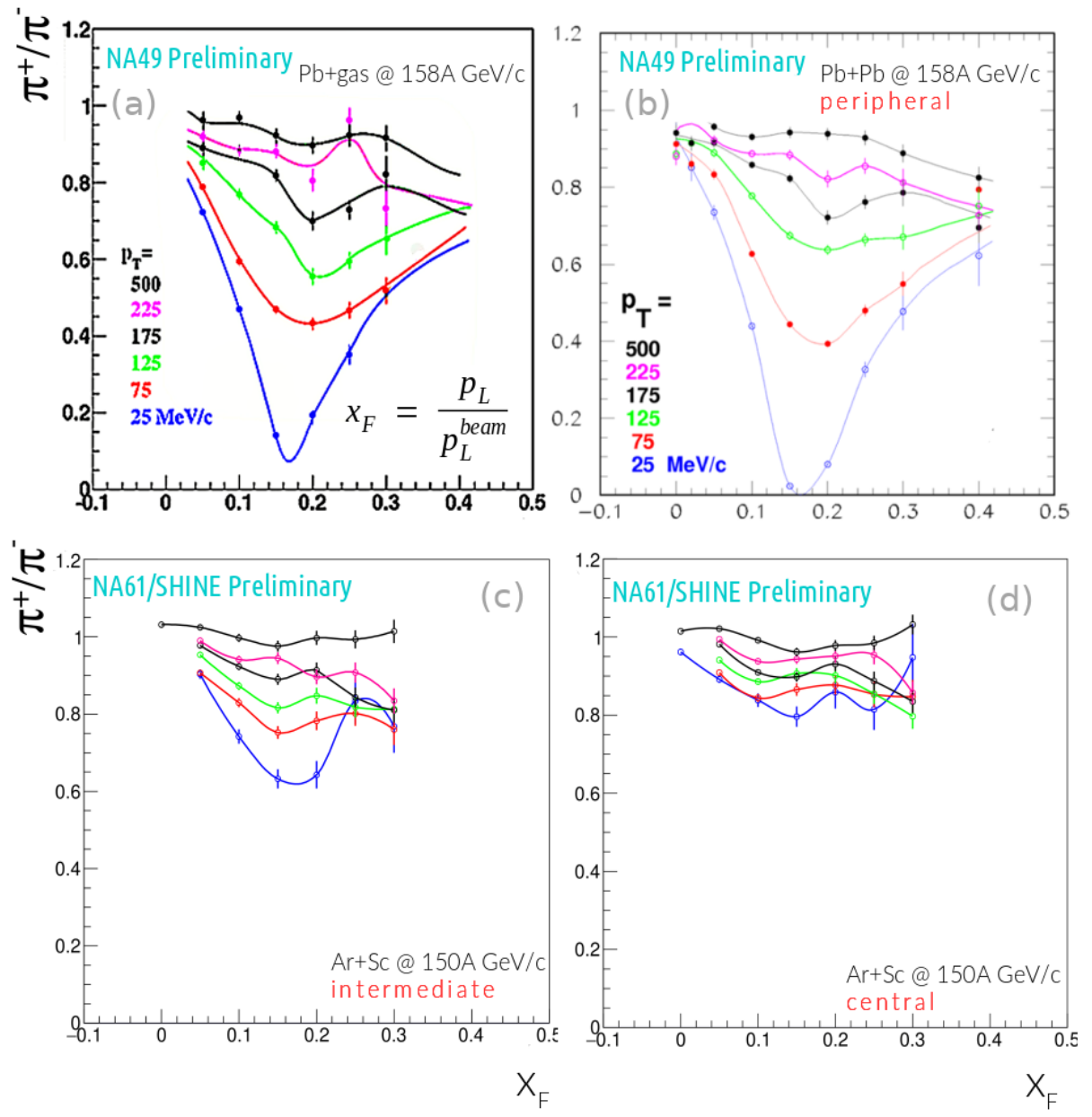

Figure 2: Panels (a)-(d) show ratios of $\pi$ mesons $\left(\pi^{+} / \pi^{-}\right)$drawn as a function of $x_{F}$ (in nucleon-nucleon c.m.s.) at several values of $p_{\mathrm{T}}$ for experimental data: (a) from $\mathrm{Pb}+$ gas collisions [5] and, (b) peripheral $\mathrm{Pb}+\mathrm{Pb}$ reactions [1] measured in the SPS NA49 experiment at $\sqrt{s_{\mathrm{NN}}}=17.3 \mathrm{GeV}$, as well as (c) intermediate and (d) central Ar+Sc collisions obtained in the SPS NA61/SHINE experiment at $\sqrt{s_{\mathrm{NN}}}=16.8 \mathrm{GeV}$ [6].

From the direct comparison of the results on EM effects obtained in three different colliding

\footnotetext{
${ }^{1}$ The Feynman variable: $x_{F}=\frac{p_{L}}{\sqrt{s_{\mathrm{NN}}}}$
} 
systems the following picture emerges:

- There are several similarities in the behavior of the data points observed between results obtained for $\mathrm{Pb}+$ gas and peripheral $\mathrm{Pb}+\mathrm{Pb}$ collisions. The resemblance is both qualitative and quantitative. In the figures, experimental data display an evident excess of the negative over positive $\pi$ mesons present for all the studied kinematic range. The moderate excess of negative over positive pions visible for the higher transverse momentum is a direct result of the isospin effect originated from the nucleonic composition of $\mathrm{Pb}$ projectile (surplus of neutrons over protons in the nucleus). For low values of $p_{\mathrm{T}}$ a characteristic structure as a function of $x_{F}$ and $p_{\mathrm{T}}$ with a sharp decrease of $\pi^{+} / \pi^{-}$ratio that breaks isospin symmetry can be seen. The observed minimum of the charged $\pi$ meson ratio, the position of which corresponds to pions moving longitudinally with the same velocity as spectator system $\left(x_{F}=m_{\pi} / m_{p}=0.15\right)$ in $\mathrm{Pb}+$ gas and peripheral $\mathrm{Pb}+\mathrm{Pb}$ collisions, is a direct result of the electromagnetic interaction between charged pions and the spectator system.

- New data on Ar+Sc collisions, bring forward the first piece of information on EM effects in small systems. Surprisingly, the EM distortion of charged $\pi$ meson ratios still remains well visible in the proximity of the $x_{F}=0.15$ for intermediate Ar+Sc data presented in Fig. 2(c). In contrast to peripheral $\mathrm{Pb}+\mathrm{Pb}$ collisions where the spectator charge of about 70 elementary units induces large lowering of $\frac{\pi^{+}}{\pi^{-}}$ratio, the observed EM effects for intermediate $\mathrm{Ar}+\mathrm{Sc}$ collisions (Fig. 2(c)) are significantly smaller at the same energy, however strong enough to break the isospin symmetry, account taken of the proportion between neutrons and protons in the ${ }_{18}^{40} \mathrm{Ar}$ nucleus. Breaking isospin symmetry takes place even if the spectator charge is around 9 times lower than in peripheral $\mathrm{Pb}+\mathrm{Pb}$ collisions.

- Observed in Fig. 2(c) for the central Ar+Sc collisions, the slight decrease of $\frac{\pi^{+}}{\pi^{-}}$ratio around beam rapidity may indicate a shadow of the EM effect still present even for a very low spectator charge.

\subsection{Phenomenological description of EM effect including spectator dynamics}

Figure 3 (a) -(f) show the results of a phenomenological study of electromagnetic effect in peripheral $\mathrm{Pb}+\mathrm{Pb}$ and intermediate $\mathrm{Ar}+\mathrm{Sc}$ collisions. Detailed information on the approach taken in the Monte Carlo simulation can be found in Ref. [1], below only a short summary of the fundamental assumptions implemented in the model is provided.

The simulation assumes that charged $\pi$ mesons are emitted from a single point and interact electromagnetically with the spectator systems. In the most basic scenario the spectator systems are modeled as two charged uniform spheres in their rest frames, they are assumed to be stable. which means that they are characterized by a zero expansion velocity $(\beta=0$, the red line in Fig. $3)$. The free parameter of the model is the distance $d_{E}$ between the $\pi$ meson freeze-out point and the spectator system at the moment of pion emission taken in collision c.m.s. Presented in Figure 3 results of MC studies were carried out for various values of distance $d_{E}$, for both peripheral $\mathrm{Pb}+\mathrm{Pb}$ collisions and intermediate $\mathrm{Ar}+\mathrm{Sc}$ reactions. From the Figure 3 it is immediately apparent that:

- Electromagnetic distortion of $\frac{\pi^{+}}{\pi^{-}}$ratios is sensitive to distance $d_{E}$ between pion emission zone and spectator systems. This is evident from comparison of the results of MC studies 


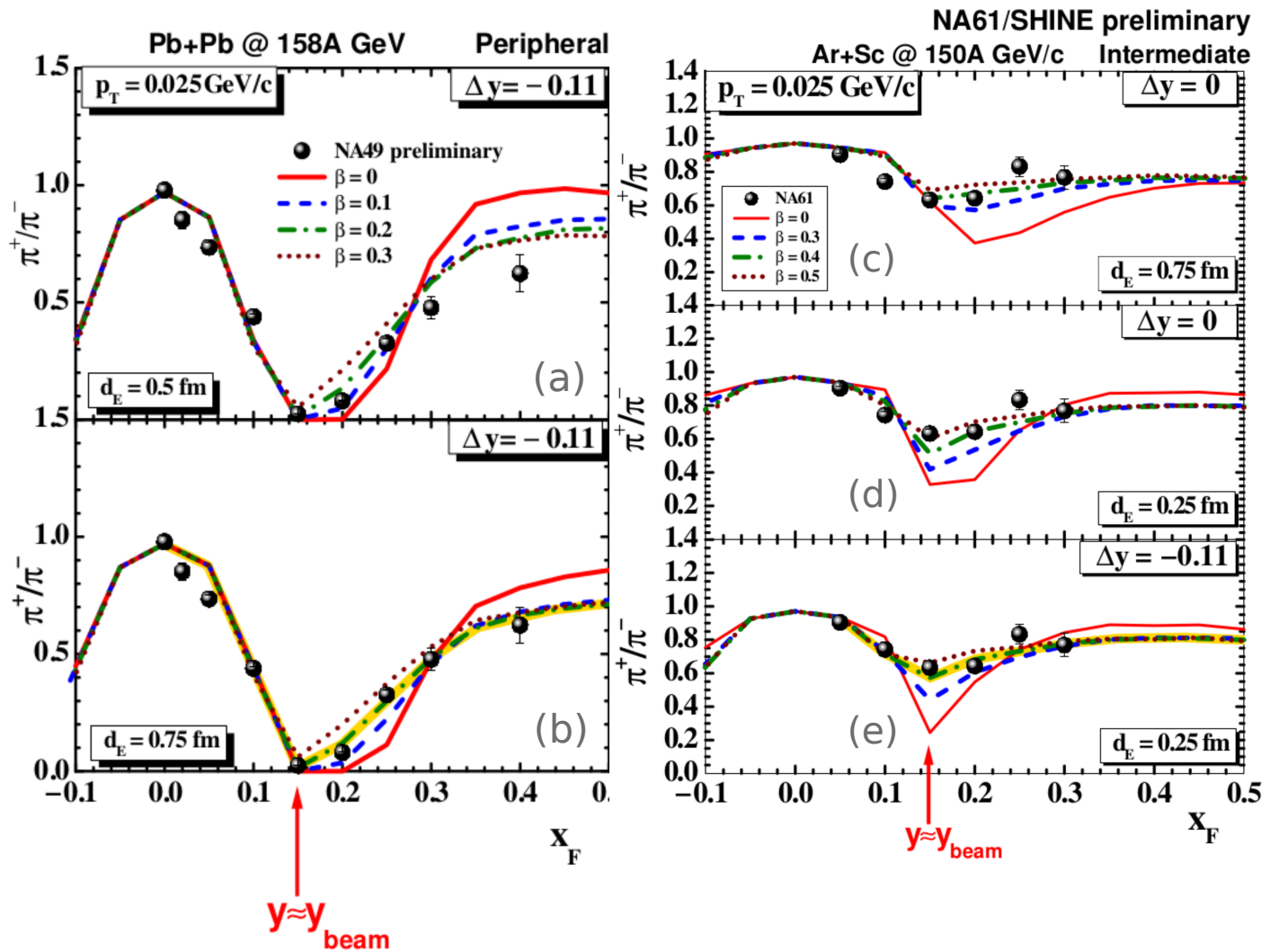

Figure 3: Panels (a)-(e) show the results of dedicated MC simulations (colour lines) of the EM distortion of $\frac{\pi^{+}}{\pi^{-}}$ratios compared to experimental results on peripheral $\mathrm{Pb}+\mathrm{Pb}$ collisions (panels (a) and (b)) and intermediate $\mathrm{Ar}+\mathrm{Sc}$ collisions (panels from (c) to (e)). MC results are obtained for different combinations of three parameters: the distance $d_{E}$, the expansion velocity $\beta$ and the rapidity shift of the effective charge cloud $\Delta y$ (more description in the text). The optimal description of experimental data is indicated by the thick solid gray/yellow line in panel (b) for peripheral $\mathrm{Pb}+\mathrm{Pb}$ collisions and in panel (e) for intermediate $\mathrm{Ar}+\mathrm{Sc}$ reactions.

obtained for two different values of distance $d_{E}$, independently for peripheral $\mathrm{Pb}+\mathrm{Pb}$ (panel (a) versus panel (b)) and intermediate $\mathrm{Ar}+\mathrm{Sc}$ collisions (panel (c) versus panel (e)).

- Contrary to the case of peripheral $\mathrm{Pb}+\mathrm{Pb}$ reactions, the non-expanding spectator system $(\beta=$ 0 ) cannot describe the $\mathrm{Ar}+\mathrm{Sc}$ experimental data. The $\mathrm{MC}$ description of the results for $\mathrm{Ar}+\mathrm{Sc}$ collisions improves if the phenomenological analysis includes spectator dynamics like e.g. expansion of the corresponding charge cloud, with significant expansion velocity $\beta>0$. The same becomes evident from a re-analysis of $\mathrm{Pb}+\mathrm{Pb}$ collisions inspired by the new data on $\mathrm{Ar}+\mathrm{Sc}$ reactions.

- In both reactions, the experimental data suggest a short longitudinal distance $d_{E}$ between the emission zone of a high $x_{F}\left(x_{F}>0.1\right)$ pion and the spectator system $\left(d_{E}<1 \mathrm{fm}\right)$.

- The optimal description is obtained by including the decrease in the rapidity of the expanding charged cloud in the model. This scenario is presented in Fig. 3 (b) for peripheral $\mathrm{Pb}+\mathrm{Pb}$ 
and in Fig. 3 (e) for intermediate Ar+Sc collisions. It could be justified in the case when the electromagnetic effect is induced by an effective charge cloud consisting of the highly charged spectator and the faster part of participant matter.

\section{Conclusions}

This paper reports a short overview of the spectator-induced electromagnetic effects observed in three different colliding systems: $\mathrm{Pb}+$ gas, peripheral $\mathrm{Pb}+\mathrm{Pb}$ and $\mathrm{Ar}+\mathrm{Sc}$ collisions at $\mathrm{CERN}$ SPS energies. New data on Ar+Sc collisions from the NA61/SHINE experiment brought a first preliminary insight into EM effects in small systems.

In summary, the findings discussed above show that the presence of EM fields in the light- and heavy-ion collision results in charge-dependent effects on various observables. The phenomenological analysis of presented experimental data sheds new light on the space-time evolution of EM effects in nucleus-nucleus collisions. The EM distortion is sensitive to the distance $d_{E}$ between the pion emission site and the spectator(s). The stable spectator scenario fails to describe the experimental results, this is most evident for a small system like intermediate $\mathrm{Ar}+\mathrm{Sc}$ collisions. The outcome of the dedicated MC simulation indicates that there is a sensitivity to the space-time evolution of the spectator system which has to be taken into account. Further research should be undertaken to investigate the precise role of the evolution of the spectator system on the measured charged particle spectra.

This work was supported by the National Science Centre, Poland (grant No. 2014/14/E/ST2/00018).

\section{References}

[1] A. Rybicki and A. Szczurek, The Spectator Electromagnetic Effect on Charged Pion Spectra in Peripheral Ultrarelativistic Heavy-Ion Collisions, Phys. Rev. C 75, 054903 (2007) doi:10.1103/PhysRevC.75.054903 [nucl-th/0610036].

[2] M. Kłusek-Gawenda, E. Kozik, A. Rybicki, I. Sputowska and A. Szczurek, Strong and Electromagnetic Forces in Heavy Ion Collisions, Acta Phys. Polon. Supp. 6, 451 (2013) doi:10.5506/APhysPolBSupp.6.451 [arXiv:1303.6423 [nucl-ex]].

[3] A. Rybicki, A. Szczurek, M. Kłusek-Gawenda, N. Davis, V. Ozvenchuk and M. Kiełbowicz, Electromagnetic effects on meson production: a new tool for studying the space-time evolution of heavy ion collisions, EPJ Web Conf. 130, 05016 (2016) doi:10.1051/epjconf/201613005016 [arXiv:1607.00413 [nucl-th]].

[4] A. Rybicki, A. Szczurek, M. Kiełbowicz, N. Davis and V. Ozvenchuk, Can we obtain a "new femtoscopy" on the basis of electromagnetic effects?, Acta Phys. Polon. Supp. 9, 303 (2016) doi:10.5506/APhysPolBSupp.9.303 [arXiv:1603.07558 [nucl-th]].

[5] I. Sputowska, A. Rybicki, The Spectator-Induced Electromagnetic Effect on Meson Production in Nucleus-Nucleus Collisions at SPS Energies, EPJ Web Conf. 37, 09035 (2012) doi:10.1051/epjconf/20123709035. 
[6] M. Kiełbowicz, Electromagnetic Effects on Charged Pion Spectra at SPS Energies, Acta Phys. Polon. B Proceedings Supp. 12, 353 (2019) doi:10.1051/epjconf/20123709035. 